\title{
APPLICATION OF MULTISTEP REPRODUCING KERNEL HILBERT SPACE METHOD FOR SOLVING GIVING UP SMOKING MODEL
}

\author{
Samia Bushnaq ${ }^{1}$, Banan Maayah ${ }^{2}$, Asma AlHabees ${ }^{3}$ \\ ${ }^{1}$ Department of Basic Sciences \\ King Abdullah II Faculty of Engineering \\ Princess Sumaya University for Technology \\ Amman 11941, JORDAN \\ ${ }^{2,3}$ Department of Mathematics \\ Faculty of Science \\ The University of Jordan \\ Amman 11942, JORDAN
}

\begin{abstract}
Smoking is the leading avoidable cause of death in the world. In this paper, we apply the Reproducing Kernel Hilbert Space method on the giving up smoking model to find an approximate solution of the model then we compare it with the fourth order Runge-Kutta method. The solutions are represented in the form of series in the Hilbert space $W_{2}^{2}[a, b]$ with easily computable components. In finding the computational solutions, we use generating the orthogonal basis from the obtained kernel functions such that the orthonormal basis is constructing in order to formulate and utilize the solutions. Numerical experiments are carried where two smooth reproducing kernel functions are used throughout the evolution of the algorithm to obtain the required nodal values of the unknown variables. The utilized graphical results show that the present algorithm and simulated annealing provide a good scheduling methodology to such model.
\end{abstract}

AMS Subject Classification: 65L05, 65L06

Key Words: reproducing kernel Hilbert space method (RKHSM), giving up smoking model, numerical solution

Received: May 9, 2016

Published: September 10, 2016
(C) 2016 Academic Publications, Ltd. url: www.acadpubl.eu 


\section{Introduction}

Smoking is a serious public-health problem with numerous effects on smokers themselves, society and family. It harms nearly every organ of the body and changes a person's overall health. For example, smoking affects the sense of taste and smell. Also, it causes throat cancer, mouth cancer, stomach cancer, lung cancer, asthma, heart disease, stroke, etc. According to the World Health Organization (WHO), nearly six million people die annually from cigarette smoking [1]. In addition, Hong Kong Special Administrative Region (HKSAR) has indicated that smoking is the leading factor of death and premature death. They also indicated that smoking is related to the top-5 diseases that would lead to death [2].

Quitting smoking reduces the risk of developing many fatal diseases. In addition, it has been reported that quitting smoking by two to five years would reduce the risk of strokes to the same extent as non-smokers. Moreover, the risks for cancers of the mouth, throat esophagus and bladder drop by half [3].

Efforts have been made in order to have a free smoking life. In this regard, many researchers are interested in understanding, exploring and explaining the spread of smoking and its effects on smoker's organs [4, 5, 6, 7, 8, 9, 10]. Many mathematical models are used to help quitting smoking among smoking persons. For example, Zaman [11] proposed a modified model that describes giving up smoking model. He studied the qualitative behavior of smoking and represented numerical simulation by using numerical methods.

The general theory of reproducing kernels was introduced by N. Aronszajn [12] and since that time, the reproducing kernel Hilbert space has played a main role in operator theory and applications. The Reproducing Kernel Hilbert Space (RKHS) method has been successfully applied to solve many types of linear, nonlinear differential and integro-differential equations $[14,15,16,17,18,19$, $20,21,22,23,24]$.

The structure of this paper is organized as follows. In Section 2, some mathematical preliminaries are introduced. In Section 3, the Reproducing Kernel Hilbert Space method (RKHSM) is proposed. In section 4, the proposed method is applied to solve the Giving Up Smoking model and numerical solutions are presented graphically. Finally, the conclusion is given in Section 5 . 


\section{Mathematical Preliminaries}

Prior to discussing the applicability of the RKHSM in solving the giving up smoking method, it is necessary to present an appropriate brief introduction to preliminary topics from the reproducing kernel theory [12].

Definition 2.1. Let $H$ be a Hilbert space of a function $f: X \rightarrow F$ on a set $X$. A Dirac functional at $x$ is a functional $\delta_{x} \in H$ such that $\delta_{x}(f)=f(x)$.

Definition 2.2. Let $\psi$ be a mapping from $X$ into the space $H$ such that $\psi(x)=K(x,$.$) . A function K: X \times X \rightarrow R$ such that $K(x, y)=$ $\langle\psi(x), \psi(y)\rangle, \forall x, y \in X$ is called a kernel.

Definition 2.3. Let $X$ be an arbitrary set. A symmetric function $K$ : $X \times X \rightarrow C$ is called positive definite kernel if $\sum_{i, j=1}^{n} \overline{\alpha_{i}} \alpha_{j} K\left(x_{i}, x_{j}\right)>0, \forall n \in$ $N, x_{1}, x_{2}, \ldots, x_{n} \in X$ and $\alpha_{i} \in C, i=1,2, \ldots$,

Definition 2.4. Let $H$ be a Hilbert space of function $f: X \rightarrow F$ on a set $X$. A function $K: X \times X \rightarrow C$ is a reproducing kernel of $H$ if the following conditions are satisfied:

1. $K(., t) \in H$ for all $t \in X$.

2. $\langle f, K(., t)\rangle=f(t)$ for all $f \in H$ for all $t \in X$.

It can be easily proved that the reproducing kernel is unique, symmetrical and positively definite.

Definition 2.5. The space $W_{2}^{m}[a, b]$ is defined by: $W_{2}^{m}[a, b]=\left\{u: u^{(j)}\right.$ are absolutely continuous functions, $j=1,2, \ldots, m-1$ and $\left.u^{(m)} \in L^{2}[a, b]\right\}$ with the inner product:

$$
\langle u, v\rangle_{W_{2}^{m}}=\sum_{i=0}^{m-1} u^{(i)}(a) v^{(i)}(a)+\int_{a}^{b} u^{(m)}(t) v^{(m)}(t) d t
$$

and norm: $\|u\|_{W_{2}^{m}}=\sqrt{\langle u, u\rangle_{W_{2}^{m}}}$. 
Theorem 2.6. The space $W_{2}^{m}[a, b]$ equipped with its norm, is a Hilbert space.

Proof. See [13].

Theorem 2.7. The function space $W_{2}^{m}[a, b]$ is a reproducing kernel space, that is, for each fixed $x \in[a, b]$ and for any $u(t) \in W_{2}^{m}[a, b], \exists K(x, t) \in W_{2}^{m}[a, b]$ such that $\langle u(t), K(x, t)\rangle=u(t)$ and $K(x, t)$ is called reproducing kernel function of $W_{2}^{m}[a, b]$.

Proof. See [12].

\section{Reproducing Kernel Hilbert Space Method (RKHSM)}

In this paper, we apply the Reproducing Kernel Hilbert Space Method (RKHSM) on a modified model that describes a giving up smoking [11]. This model is given by:

$$
\begin{aligned}
\frac{d P}{d t} & =b M(t)-\beta_{1}(t) L(t) P(t)-\left(d_{1}+\mu\right) P(t)+\tau Q(t), \\
\frac{d L}{d t} & =\beta_{1}(t) L(t) P(t)-\beta_{2}(t) L(t) S(t)-\left(d_{2}+\mu\right) L(t), \\
\frac{d S}{d t} & =\beta_{2}(t) L(t) S(t)-\left(\gamma+d_{3}+\mu\right) S(t), \\
\frac{d Q}{d t} & =\gamma S(t)-\left(\tau+d_{4}+\mu\right) Q(t), \\
\frac{d M}{d t} & =(b-\mu) M(t)-\left(d_{1} P(t)+d_{2} L(t)+d_{3} S(t)+d_{4} Q(t)\right),
\end{aligned}
$$

with initial conditions:

$$
P(0)=c_{1}, L(0)=c_{2}, S(0)=c_{3}, Q(0)=c_{4}, M(0)=c_{5}
$$

where $P(t), L(t), S(t), Q(t)$ and $M(t)$ denote the numbers of potential smokers, occasional smokers, smokers, quit smokers and total smokers at time $t$, respectively. Here, $b$ is the birth rate, $\mu$ is the natural death rate, $\gamma$ is the recovery rate from smoking, $\beta_{1}(t)$ and $\beta_{2}(t)$ are transmission coefficients, $d_{1}, d_{2}, d_{3}$, and $d_{4}$ are death rates of potential smokers, occasional smokers, smokers and quit smokers related to smoking disease, respectively. Additionally, $\tau$ represents the rate at which a quit smoker in the population becomes a potential smoker again. 
The Giving Up Smoking Model has been studied by many authors [25, 26, 27]. In this article, we try to employ the Reproducing Kernel Hilbert Space Method (RKHSM) on this model in order to find an approximate solution and compare it with the forth order classical Runge-Kutta method.

First, we need to homogenize the initial conditions of the model (3.1 - 3.6) with the following transformations:

$$
\begin{gathered}
p(t)=P(t)-c_{1}, l(t)=L(t)-c_{2}, s(t)=S(t)-c_{3}, \\
q(t)=Q(t)-c_{4} \text { and } m(t)=M(t)-c_{5} .
\end{gathered}
$$

By these transformations, the system (3.1 - 3.6) can be written as:

$$
\begin{aligned}
\frac{d p}{d t}= & b\left(m(t)+c_{5}\right)-\beta_{1}(t)\left(l(t)+c_{2}\right)\left(p(t)+c_{1}\right)-\left(d_{1}+\mu\right)\left(p(t)+c_{1}\right) \\
& +\tau\left(q(t)+c_{4}\right) \\
\frac{d l}{d t}= & \beta_{1}(t)\left(l(t)+c_{2}\right)\left(p(t)+c_{1}\right)-\beta_{2}(t)\left(l(t)+c_{2}\right)\left(s(t)+c_{3}\right) \\
& -\left(d_{2}+\mu\right)\left(l(t)+c_{2}\right) \\
\frac{d s}{d t}= & \beta_{2}(t)\left(l(t)+c_{2}\right)\left(s(t)+c_{3}\right)-\left(\gamma+d_{3}+\mu\right)\left(s(t)+c_{3}\right) \\
\frac{d q}{d t}= & \gamma\left(s(t)+c_{3}\right)-\left(\tau+d_{4}+\mu\right)\left(q(t)+c_{4}\right) \\
\frac{d m}{d t}= & (b-\mu)\left(m(t)+c_{5}\right)-\left(d_{1}\left(p(t)+c_{1}\right)+d_{2}\left(l(t)+c_{2}\right)\right. \\
& \left.+d_{3}\left(s(t)+c_{3}\right)+d_{4}\left(q(t)+c_{4}\right)\right)
\end{aligned}
$$

with the initial conditions:

$$
p(0)=l(0)=s(0)=q(0)=m(0)=0
$$

Second, we construct reproducing kernel for the spaces $W_{2}^{1}[a, b]$ and $W_{2}^{2}[a, b]$ in which every function satisfies the homogenous initial conditions (3.12).

- [17] The inner product space $W_{2}^{1}[a, b]=\{u \mid u$ is an absolutely continuous real valued function, $\left.u^{\prime} \in L^{2}[a, b]\right\}$ with the inner product

$$
\langle u, v\rangle_{W_{2}^{1}}=\int_{a}^{b}\left(u(t) v(t)+u^{\prime}(t) v^{\prime}(t)\right) d t
$$

is a complete reproducing kernel and its reproducing kernel is given by:

$$
R(x, y)=\frac{1}{\sinh (b-a)}[\cosh (x+y-b-a)+\cosh (|x-y|-b-a)]
$$


- [18] The inner product space $W_{2}^{2}[a, b]=\left\{u \mid u, u^{\prime}\right.$ are absolutely continuous real valued functions, $\left.u^{\prime \prime} \in L^{2}[a, b], u(a)=0\right\}$ with the inner product

$$
\langle u, v\rangle_{W_{2}^{2}}=u(a) v(a)+u^{\prime}(a) v^{\prime}(a)+\int_{a}^{b} u^{\prime \prime}(t) v^{\prime \prime}(t) d t
$$

is a complete reproducing kernel and its reproducing kernel is given by:

$$
K(x, y)=\frac{-1}{6} \begin{cases}(a-y)\left(2 a^{2}-y^{2}+3 x(2+y)-a(6+3 x+y)\right) & \text { if } y \leq x \\ (a-x)\left(2 a^{2}-x^{2}+3 y(2+x)-a(6+3 y+x)\right) & \text { if } y>x\end{cases}
$$

Now, we define the bounded linear operators $T_{i}: W_{2}^{2}[a, b] \rightarrow W_{2}^{1}[a, b], i=$ $1,2, \ldots, 5$ such that:

$$
\begin{aligned}
T_{1} p(t)= & \frac{d p}{d t}=b\left(m(t)+c_{5}\right)-\beta_{1}(t)\left(l(t)+c_{2}\right)\left(p(t)+c_{1}\right) \\
& -\left(d_{1}+\mu\right)\left(p(t)+c_{1}\right)+\tau\left(q(t)+c_{4}\right), \\
T_{2} l(t)= & \frac{d l}{d t}=\beta_{1}(t)\left(l(t)+c_{2}\right)\left(p(t)+c_{1}\right) \\
& -\beta_{2}(t)\left(l(t)+c_{2}\right)\left(s(t)+c_{3}\right)-\left(d_{2}+\mu\right)\left(l(t)+c_{2}\right), \\
T_{3} s(t)= & \frac{d s}{d t}=\beta_{2}(t)\left(l(t)+c_{2}\right)\left(s(t)+c_{3}\right)-\left(\gamma+d_{3}+\mu\right)\left(s(t)+c_{3}\right), \\
T_{4} q(t)= & \frac{d q}{d t}=\gamma\left(s(t)+c_{3}\right)-\left(\tau+d_{4}+\mu\right)\left(q(t)+c_{4}\right), \\
T_{5} m(t)= & \frac{d m}{d t}=(b-\mu)\left(m(t)+c_{5}\right) \\
& -\left(d_{1}\left(p(t)+c_{1}\right)+d_{2}\left(l(t)+c_{2}\right)+d_{3}\left(s(t)+c_{3}\right)+d_{4}\left(q(t)+c_{4}\right)\right) .
\end{aligned}
$$

The next step is to construct an orthogonal function system of $W_{2}^{2}[a, b]$.

To do this, let $\left\{t_{j}\right\}_{j=1}^{\infty}$ be a countable dense set in $[a, b]$. Let $\varphi_{j}^{i}(t)=R\left(t_{j}, t\right)$ and $\psi_{j}^{i}(t)=T_{i}^{*} \varphi_{j}^{i}(t), i=1, \ldots, 5$; where $T_{i}^{*}$ is the self-adjoint operator of $T_{i}, i=$ $1, \ldots, 5$.

The orthonormal system $\left\{\bar{\psi}_{j}^{i}(t)\right\}_{j=1}^{\infty}$ of the space $W_{2}^{2}[a, b]$ can be determined by Gram- Schmidt orthogonalization process of

$$
\left\{\psi_{j}^{i}(t)\right\}_{j=1}^{\infty}, \bar{\psi}_{j}^{i}(t)=\sum_{s=1}^{j} \eta_{j k}^{i} \psi_{k}^{i}(t), \quad j=1,2, \ldots
$$

where $\eta_{j k}^{i}, i=1, \ldots, 5$ are the orthogonalization coefficients. 
Theorem 3.1. Assume that the inverse operator $T_{i}^{-1}$ exists. Then if $\left\{t_{j}\right\}_{j=1}^{\infty}$ is dense on $[a, b]$, then $\left\{\psi_{j}^{i}(t)\right\}_{j=1}^{\infty}, i=1, \ldots, 5$ is a complete system of $W_{2}^{2}[a, b]$.

Proof. Let $u(t) \in W_{2}^{2}[a, b]$ such that $\left\langle u(t), \psi_{j}^{i}(t)\right\rangle_{W_{2}^{2}}=0, \forall j=1,2, \ldots$ Then

$$
0=\left\langle u(t), \psi_{j}^{i}(t)\right\rangle_{W_{2}^{2}}=\left\langle u(t), T_{i}^{*} \varphi_{j}^{i}(t)\right\rangle_{W_{2}^{2}}=\left\langle T_{i} u(t), R\left(t_{j}, t\right)\right\rangle_{W_{2}^{2}}=T_{i} u\left(t_{j}\right) .
$$

Since $\left\{t_{j}\right\}_{j=1}^{\infty}$ is dense on $[a, b]$, then $T_{i} u\left(t_{j}\right)=0 \Rightarrow u \equiv 0$ since $T_{i}^{-1}$ exists.

Theorem 3.2. If $\left\{t_{j}\right\}_{j=1}^{\infty}$ is dense on $[a, b]$ and the solution of equation (3.1 - 3.6) is unique, then the solution is given by:

$$
\begin{aligned}
& P(t)=\sum_{j=1}^{\infty} A_{j} \bar{\psi}_{j}^{1}(t)+c_{1} ; \text { where } \\
& A_{j}=\sum_{j=1}^{\infty} \eta_{j k}^{1}\left(b\left(m\left(t_{k}\right)+c_{5}\right)-\beta_{1}\left(t_{k}\right)\left(l\left(t_{k}\right)+c_{2}\right)\left(p\left(t_{k}\right)+c_{1}\right)\right. \\
& \left.-\left(d_{1}+\mu\right)\left(p\left(t_{k}\right)+c_{1}\right)+\tau\left(q\left(t_{k}\right)+c_{4}\right)\right), \\
& L(t)=\sum_{j=1}^{\infty} B_{j} \bar{\psi}_{j}^{2}(t)+c_{2} ; \text { where } \\
& B_{j}=\sum_{j=1}^{\infty} \eta_{j k}^{2}\left(\beta_{1}\left(t_{k}\right)\left(l\left(t_{k}\right)+c_{2}\right)\left(p\left(t_{k}\right)+c_{1}\right)\right. \\
& -\beta_{2}\left(t_{k}\right)\left(l\left(t_{k}\right)+c_{2}\right)\left(s\left(t_{k}\right)+c_{3}\right)-\left(d_{2}+\mu\right)\left(l\left(t_{)}+c_{2}\right)\right), \\
& S(t)=\sum_{j=1}^{\infty} C_{j} \bar{\psi}_{j}^{3}(t)+c_{3} ; \text { where } \\
& C_{j}=\sum_{j=1}^{\infty} \eta_{j k}^{3}\left(\beta_{2}\left(t_{k}\right)\left(l\left(t_{k}\right)+c_{2}\right)\left(s\left(t_{k}\right)+c_{3}\right)-\left(\gamma+d_{3}+\mu\right)\left(s\left(t_{k}\right)+c_{3}\right)\right), \\
& Q(t)=\sum_{j=1}^{\infty} D_{j} \bar{\psi}_{j}^{4}(t)+c_{4} ; \text { where } \\
& D_{j}=\sum_{j=1}^{\infty} \eta_{j k}^{4}\left(\gamma\left(s\left(t_{k}\right)+c_{3}\right)-\left(\tau+d_{4}+\mu\right)\left(q\left(t_{k}\right)+c_{4}\right)\right) \text {, } \\
& M(t)=\sum_{j=1}^{\infty} E_{j} \bar{\psi}_{j}^{5}(t)+c_{5} ; \text { where }
\end{aligned}
$$




$$
\begin{aligned}
E_{j}= & \sum_{j=1}^{\infty} \eta_{j k}^{5}\left((b-\mu)\left(m\left(t_{k}\right)+c_{5}\right)-\left(d_{1}\left(p\left(t_{k}\right)+c_{1}\right)\right.\right. \\
& \left.\left.+d_{2}\left(l\left(t_{k}\right)+c_{2}\right)+d_{3}\left(s\left(t_{k}\right)+c_{3}\right)+d_{4}\left(q\left(t_{k}\right)+c_{4}\right)\right)\right) .
\end{aligned}
$$

Proof. $\left\{\bar{\psi}_{j}^{1}(t)\right\}_{j=1}^{\infty}$ is a complete orthonormal basis of $W_{2}^{2}[a, b]$,

$$
\begin{aligned}
p(t)= & \sum_{j=1}^{\infty}\left\langle p(t), \bar{\psi}_{j}^{1}(t)\right\rangle_{W_{2}^{2}} \bar{\psi}_{j}^{1}(t)=\sum_{j=1}^{\infty}\left\langle p(t), \sum_{k=1}^{j} \eta_{j k}^{1} \psi_{k}^{1}(t)\right\rangle_{W_{2}^{2}} \bar{\psi}_{j}^{1}(t) \\
= & \sum_{j=1}^{\infty} \sum_{k=1}^{j} \eta_{j k}^{1}\left\langle p(t), \psi_{k}^{1}(t)\right\rangle_{W_{2}^{2}} \bar{\psi}_{j}^{1}(t)=\sum_{j=1}^{\infty} \sum_{k=1}^{j} \eta_{j k}^{1}\left\langle p(t), T_{1}^{*} \varphi_{k}^{1}(t)\right\rangle_{W_{2}^{2}} \bar{\psi}_{j}^{1}(t) \\
= & \sum_{j=1}^{\infty} \sum_{k=1}^{j} \eta_{j k}^{1}\left\langle T_{1} p(t), \varphi_{k}^{1}(t)\right\rangle_{W_{2}^{1}} \bar{\psi}_{j}^{1}(t)=\sum_{j=1}^{\infty} \sum_{k=1}^{j} \eta_{j k}^{1}\left\langle T_{1} p(t), R\left(t_{k}, t\right)\right\rangle_{W_{2}^{1}} \bar{\psi}_{j}^{1}(t) \\
= & \sum_{j=1}^{\infty} \sum_{k=1}^{j} \eta_{j k} T_{1} p\left(t_{k}\right) \bar{\psi}_{j}^{1}(t) \\
= & \sum_{j=1}^{\infty} \sum_{k=1}^{j} \eta_{j k}^{1}\left(b\left(m\left(t_{k}\right)+c_{5}\right)-\beta_{1}\left(t_{k}\right)\left(l\left(t_{k}\right)+c_{2}\right)\left(p\left(t_{k}\right)+c_{1}\right)\right. \\
& \left.-\left(d_{1}+\mu\right)\left(p\left(t_{k}\right)+c_{1}\right)+\tau\left(q\left(t_{k}\right)+c_{4}\right)\right) \bar{\psi}_{j}^{1}(t) \\
= & \sum_{j=1}^{\infty} A_{j} \bar{\psi}_{j}^{1}(t) .
\end{aligned}
$$

Therefore, $P(t)=p(t)+c_{1}$.

Similarly, we can prove the result for $L(t), S(t), Q(t)$ and $M(t)$.

The approximate solution can be obtained by taking finitely many terms in the series representation:

$$
\begin{gathered}
P_{n}(t)=\sum_{j=1}^{n} A_{j} \bar{\psi}_{j}^{1}(t)+c_{1}, L_{n}(t)=\sum_{j=1}^{n} B_{j} \bar{\psi}_{j}^{2}(t)+c_{2} \\
S_{n}(t)=\sum_{j=1}^{n} C_{j} \bar{\psi}_{j}^{3}(t)+c_{3}, Q_{n}(t)=\sum_{j=1}^{n} D_{j} \bar{\psi}_{j}^{4}(t)+c_{4}, M_{n}(t)=\sum_{j=1}^{n} E_{j} \bar{\psi}_{j}^{5}(t)+c_{5} .
\end{gathered}
$$

Note that since $W_{2}^{2}[a, b]$ is a Hilbert space, then the previous series are convergent in the sense of the norm of $W_{2}^{2}[a, b]$. 


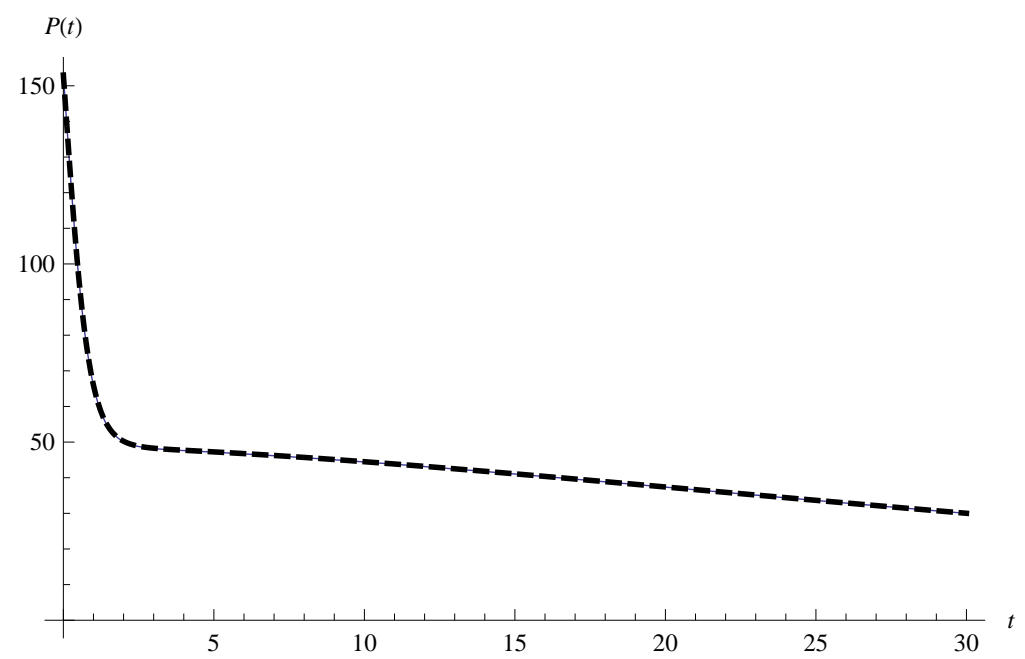

Figure 1: The Potential Smokers Individual $P(t)$

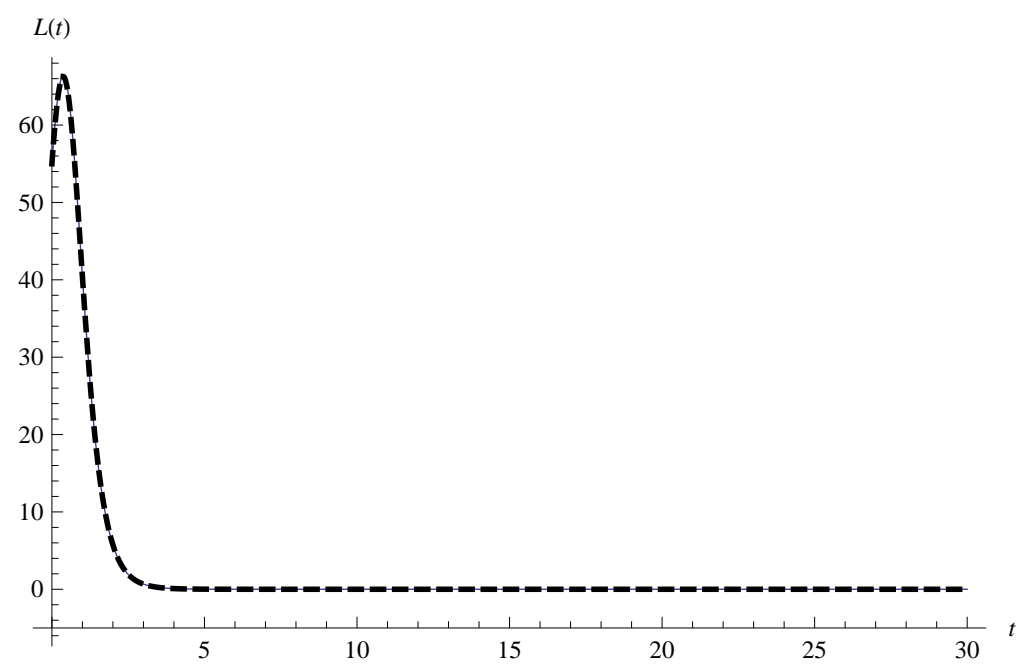

Figure 2: The Occasional Smokers Individual $L(t)$

Now, we will use the multistep RKHSM [19] to approximate the solution of the Giving Up Smoking model over $[0, b]$. We will divide the interval $[0, b]$ into $V$ subintervals $\left[t^{v-1}, t^{v}\right], v=1,2, \ldots, K$ with equal size $h=\frac{b}{V}$ and nodes $t^{v}=v h$.

$\boldsymbol{S T E P}(1):$ we apply the RKHSM to the system $(3.1-3.6), t \in\left[0, t^{1}\right]$ to get 


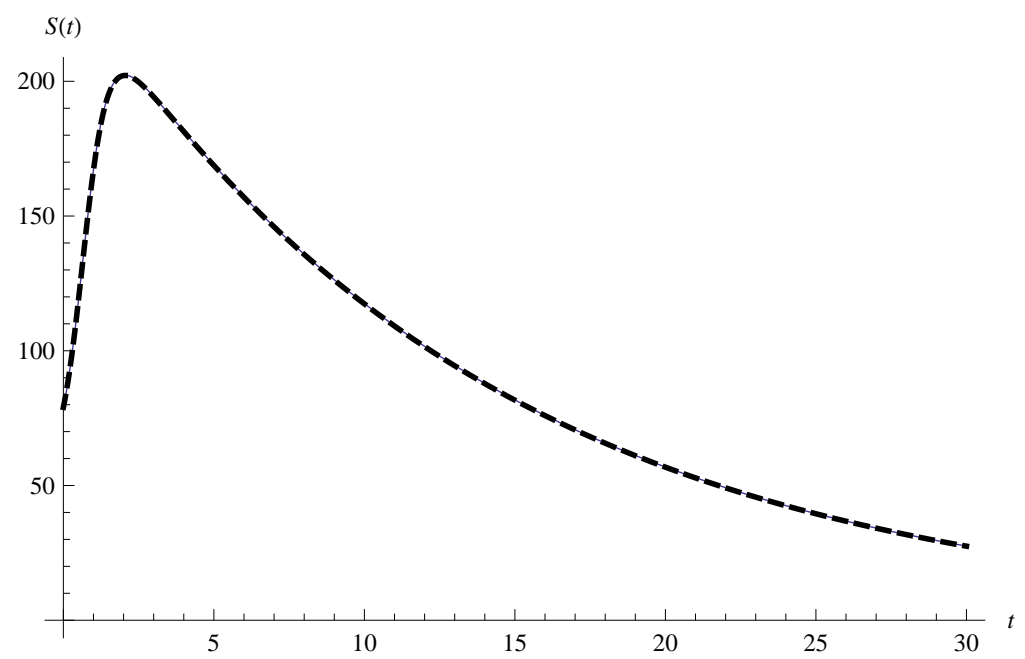

Figure 3: The Smokers Individual $S(t)$

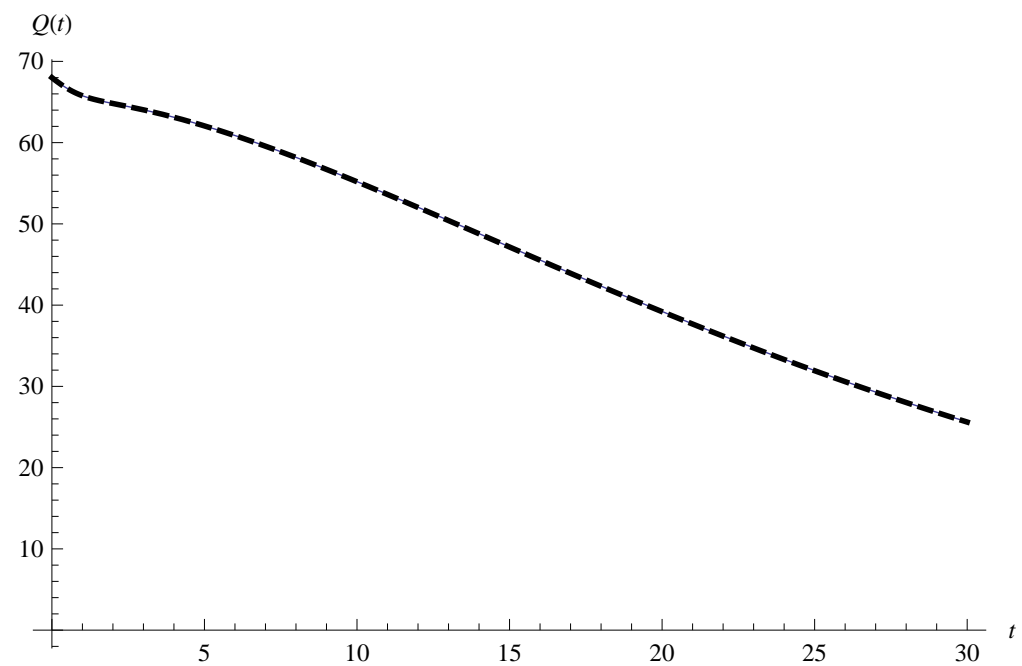

Figure 4: The Quit Smokers Individual $Q(t)$

the approximate solution $P_{1 n}(t)=\sum_{j=1}^{n} A_{1 j} \bar{\psi}_{j}^{1}(t)+c_{1}, L_{1 n}(t)=\sum_{j=1}^{n} B_{1 j} \bar{\psi}_{j}^{2}(t)+$ $c_{2}, S_{1 n}(t)=\sum_{j=1}^{n} C_{1 j} \bar{\psi}_{j}^{3}(t)+c_{3}, Q_{1 n}(t)=\sum_{j=1}^{n} D_{1 j} \bar{\psi}_{j}^{4}(t)+c_{4}, M_{1 n}(t)=$ $\sum_{j=1}^{n} E_{1 j} \bar{\psi}_{j}^{5}(t)+c_{5}$.

$\boldsymbol{S T E P}(\mathscr{2}):$ For $v \geq 2$ and subinterval $\left[t^{v-1}, t^{v}\right]$, we will use the initial conditions $P_{v}\left(t^{v-1}\right)=P_{v-1}\left(t^{v-1}\right)=\omega_{v}, L_{v}\left(t^{v-1}\right)=L_{v-1}\left(t^{v-1}\right)=\omega_{v}, S_{v}\left(t^{v-1}\right)=$ 


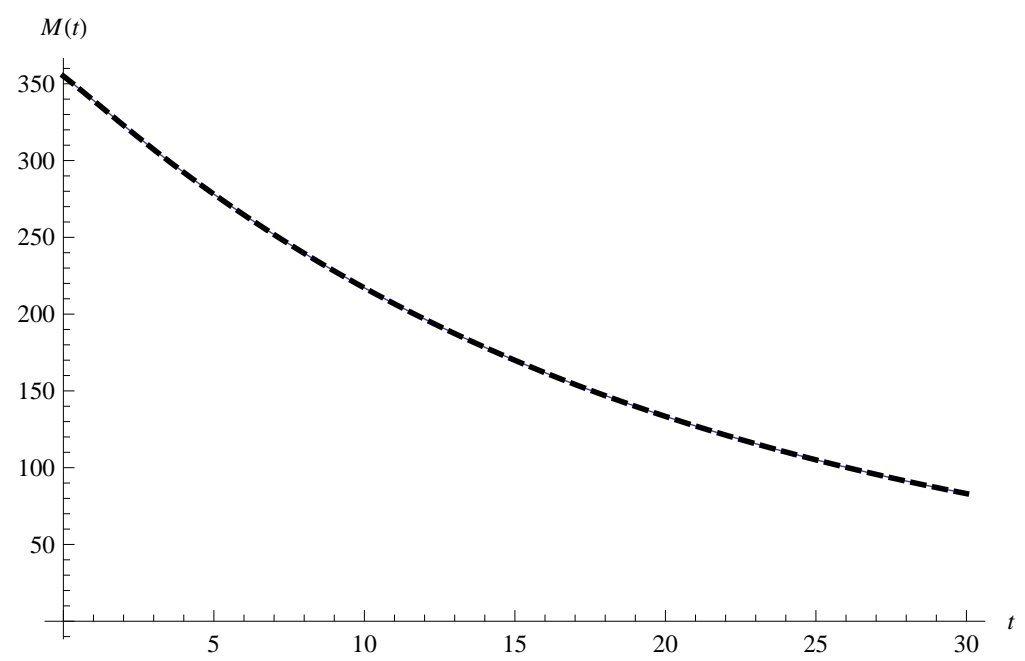

Figure 5: The Total Population $M(t)$

$S_{v-1}\left(t^{v-1}\right)=\omega_{v}, Q_{v}\left(t^{v-1}\right)=Q_{v-1}\left(t^{v-1}\right)=\omega_{v}, M_{v}\left(t^{v-1}\right)=M_{v-1}\left(t^{v-1}\right)=\omega_{v}$ and again apply the RKHSM over the interval $\left[t^{v-1}, t^{v}\right]$.

This procedure will generate a sequence of approximate solution $P_{v n}(t)$, $L_{v n}(t), S_{v n}(t), Q_{v n}(t), M_{v n}(t), v=1,2, \ldots, V$ as follows:

$$
P_{n}(t)= \begin{cases}P_{1 n}(t)=\sum_{j=1}^{n} A_{1 j} \bar{\psi}_{j}^{1}(t)+c_{1} & \text { if } t \in\left[0, t^{1}\right] \\ P_{2 n}(t)=\sum_{j=1}^{n} A_{2 j} \bar{\psi}_{j}^{1}(t)+\omega_{2} & \text { if } t \in\left[t^{1}, t^{2}\right] \\ \cdot & \\ \cdot & \\ P_{V n}(t)=\sum_{j=1}^{n} A_{V j} \bar{\psi}_{j}^{1}(t)+\omega_{V} & \text { if } t \in\left[t^{V-1}, t^{V}\right]\end{cases}
$$

We can write $L_{n}(t), S_{n}(t), Q_{n}(t)$ and $M_{n}(t)$ in the same way.

\section{Numerical Method and Simulation}

In order to solve the giving up smoking to show behavior, properties, efficiency, and applicability of the present RKHSM, the system (3.1 - 3.6) was solved using RKHSM and classical Runge Kutta method. The numerical solution of the system was obtained using the following parameters,

$$
b=0.0045, \beta_{1}=0.014, \beta_{2}=0.014, \gamma=0.0165,
$$




$$
\mu=0.0021, \tau=0, d_{1}=0.034, d_{2}=0.045, d_{3}=0.054, d_{4}=0.061,
$$

and initial conditions: $c_{1}=153, c_{2}=55, c_{3}=79, c_{4}=68, c_{5}=355$.

The Figures 1-5 show the approximate solution of the system $(3.1-3.6)$ using the RKHS and RK4 methods of $P(t), L(t), S(t), Q(t)$ and $M(t)$. From the result of the figures, we can see that the results obtained from RKHSM are similar to the results obtained from RK4.

\section{Conclusion}

In this article, the Reproducing Kernel Hilbert Space Method (RKHSM) has been successfully employed to find an approximate solution for Giving Up Smoking model. The results are compared with the results obtained by fourth order Runge-Kutta method. From the graphical results, we can conclude that the RKHSM is an efficient method for solving this kind of models. It is worth mentioning here that the algorithm is capable of reducing the volume of the computational work and complexity while still maintaining the high accuracy of the numerical results.

\section{References}

[1] World Health Organization, WHO Report on the Global Tobacco Epidemic, Geneva, Switzerland, (2011).

[2] Health Department of Hong Kong Special Administrative Region, Hong Kong Health Statistics 2009, Hong Kong, (2009).

[3] U.S. Department of Health and human Services, How Tobacco Smoke Causes Disease: What it means to you, Atlanta: U.S., (2010).

[4] L.F Stead, R. Perera, C. Bullen, et al., Nicotine Replacement Therapy for Smoking Cessation, Cochrane Database of Systematic Reviews, 11, (2012), CD000146. doi: 10.1002/14651858.CD000146.pub4 pmid:23152200..

[5] T. Boardman, D. Catlnoey, M.S. Mayo and J.S. Ahluwalia, Self-Efficiency and Motivation to Quit During Participation in a Smoking Cessation Program, International Journal of Behavioral Medicine, 12, No.4, (2005), 266-272.

[6] F.C. Bandiera, A.K. Richardson, D.J. Lee, J.P. He and K.R. Merikangas, Secondhand Smoke Exposure and Mental Health Among Children and Adolescents, Archives of Pediatrics and Adolescent Medicine, 165, No.4, (2011), 332-338.

[7] S.P. Doherty, J. Grabowski, C. Hoffman, S.P. Ng and J.T. Zelikoff, Early life Insult from Cigarette Smoke may be Predicitive of Chronic Disease Later in Life, Biomarkers, 14, No.1, (2009), 97-101.

[8] O.N. Niedermaier, M.L. Smith, L.A. Beightol, Z. Zukowska-Grojec, D.S. Goldstein and D.L. Eckberg, Influence of Cigarette Smoking on Human Autonomic Function, Circulation, 88, No.2, (1993), 562-571. 
[9] J.E. Harris, Cigarette Smoke Components and Disease: cigarette smoke is more than a triad of tar, nicotine, and carbon monoxide, NCI Smoking and Tobacco Control Monographs, Chapter 5, (1996), 59-75.

[10] G.J. Hankey, Smoking and Risk of Stroke, Journal of Cardiovascular Risk, 6, No.4, (1999), 207-211, doi.org/10.1177/204748739900600403.

[11] G. Zaman and S. Islam, A Non-Standard Numerical Method for a Giving-Up Smoking Model, Nonlinear Science Letters A, 1, No.4, (2010), 397-402.

[12] N. Aronszajn, Theory of reproducing kernels, Transactions of the American Mathematical Society, 68, No.3, (1950), 337-404, doi.org/10.1090/S0002-9947-1950-0051437-7.

[13] L. Yang and Y. Lin, Reproducing Kernel Methods for Solving Linear Initial-BoundaryValue Problems, Electronic Journal of Differential Equations, 2008, No.29, (2008), 1-11.

[14] S. Bushnaq, S. Momani and Y. Zhou, A reproducing kernel Hilbert space method for solving integro-differential equation of fractional order, Journal of Optimization Theory and Applications, 156, No.1, (2012), 90-1055, doi.org/10.1007/s10957-012-0207-2.

[15] S. Bushnaq, B. Maayah, S. Momani and A. Alsaedi, A reproducing kernel Hilbert space method for solving systems of fractional integro-differential equations, Abstract and Applied Analysis, 2014, (2014), Article ID 103016.

[16] Z. Chen and Y.Z. Lin, The exact solution of a linear integral equation with weakly singular kernel, Journal of Mathematical Analysis and Applications, 344, No.2, (2008), 726-734.

[17] M.G. Cui and C.L. Li, The exact solution for solving a class nonlinear operator equations in the reproducing kernel space, Applied Mathematics Computation, 143, (2003), 393399.

[18] M.G. Cui and Y.Z. Lin, Nonlinear Numerical Analysis in Reproducing Kernel Hilbert Space, Nova Science, New York, 2009, (2009).

[19] B. Maayah, S. Bushnaq, S. Momani and Abu Arqub, O., Iterative multistep reproducing kernel Hilbert space method for solving strongly nonlinear oscillators, Advances in Mathematical Physics, 2014, (2014), Article ID 758195.

[20] Y.Z. Lin and Y.F. Zhou, Solving nonlinear pseudoparabolic equations with nonlocal boundary conditions in reproducing kernel space, Numerical Algorithms, 52, (2009), 173-186.

[21] O. Abu Arqub, M. Al-Smadi and S. Momani, Applications of Reproducing Kernel Method for Solving Nonlinear Fredholm-Voltera Integro-Differential Equations, Abstract and Applied Analysis, 2012, (2012), Article ID: 839836.

[22] O. Abu Arqub, M. Al-Smadi and N. Shawagfeh, Solving Fredholm Integro-Differential Equations Using Reproducing Kernel Hilbert Space Method, Applied Mathematics and Computation, 219, (2013), 8938-8948.

[23] M. Al-Smadi, O. Abo Arqub and A. El-Ajuo, A Numerical Method for Solving Systems of First-Order Periodic Boundary Value Problems, Journal of Applied Mathematics, 2014, (2014), Article ID 135465, doi.org/10.1155/2014/135465.

[24] O. Abu Arqub, Reproducing Kernel Algorithm for Analytical-Numerical Solutions of Nonlinear Systems of Singular Periodic Boundary Value Problems, Mathematical Problems in Engineering, 2015, (2015), Article ID 518406, doi.org/10.1155/2015/518406.

[25] V.S. Erturk, S. Momani and G. Zaman, A numeric-analytic method for approximating a giving up smoking model containing fractional derivatives, Computers and Mathematics with Applications, 64, No.10, (2012), 3065-3074. 
[26] A. Zeb, M.I. Chohan and G. Zaman, The Homotopy Analysis Method for Approximating of Giving Up Smoking Model in Fractional Order, Applied Mathematics, 3, (2012), 914919.

[27] A. Zeb, G. Zaman and S. Momani, Square-root dynamics of a giving up smoking model, Applied Mathematical Modelling, 37, (2013), 5326-5334. 